\title{
Performance improvement of tilting-pad journal bearings by means of controllable lubrication
}

\author{
Alejandro Cerda Varela ${ }^{a}$ and Ilmar Ferreira Santos \\ Department of Mechanical Engineering, Technical University of Denmark, 2800 Kgs. Lyngby, Denmark
}

Received 28 July 2011, Accepted 3 November 2011

\begin{abstract}
Tilting-Pad Journal Bearings (TPJB) are commonly used on high-performance turbomachinery due to their excellent stability properties at high speed when compared to other designs for oil film bearings. Hence, efforts have been made to improve the accuracy for the available models for these mechanical devices, achieving nowadays an elasto-thermo-hydrodynamic formulation. On the other hand, the basic design of the Tilting-Pad Journal Bearing has been modified in order to transform it into a smart machine element. One approach to do so is to inject pressurized oil directly into the bearing clearance through holes drilled across the bearing pads. By adjusting the injection pressure, it is possible to modify the dynamic characteristics of the bearing. A controllable lubrication regime is obtained, allowing to expand the operational boundaries of the original design. This work focuses on presenting an elasto-thermo-hydrodynamic model (ETHD) for the Tilting-Pad Journal Bearing, including the effect of the controllable lubrication system. The basic model is validated by comparing its results against theoretical and experimental results available in the literature. Then, the validated code is used to show the benefits of applying a controllable lubrication regime, by means of the modification of the thermal and dynamic behaviour of the bearing.
\end{abstract}

Key words: Bearing / tilting pad / modelling / controllable / thermal / dynamics

\begin{tabular}{|ll}
\hline \multicolumn{2}{|l|}{ Nomenclature } \\
$\alpha_{0}$ & TPJB pad apperture angle ${ }^{\circ}$ ) \\
$\Delta s$ & TPJB pad thickness (m) \\
$\boldsymbol{D}_{r}$ & proportional damping matrix of the rotor \\
& finite-element model \\
$\boldsymbol{D}_{g}$ & damping matrix of the rotor-TPJBs model \\
$\mu$ & oil film dynamic viscosity (Pa.s) \\
$\mu_{\text {inj }}$ & oil injection dynamic viscosity (Pa.s) \\
$\nu$ & TPJB pad material Poisson ratio \\
$\Omega$ & rotor rotational speed (rad.s $\left.{ }^{-1}\right)$ \\
$\bar{x}$ & pad local Cartesian coordinate system \\
$\bar{y}$ & pad local Cartesian coordinate system \\
$\bar{z}$ & pad local Cartesian coordinate system \\
$\rho$ & oil density (kg.m $\left.{ }^{-3}\right)$ \\
$\rho_{s}$ & TPJB pad material density (kg.m $\left.{ }^{-3}\right)$ \\
$F$ & positioning function for injection holes $\left(\mathrm{m}^{2}\right)$ \\
$C_{p}$ & oil specific heat (J.kg ${ }^{-1}$.K) \\
$d_{0}$ & diameter of the oil injection hole (m) \\
$f_{g}$ & load vector for the rotor-TPJBs model \\
$f_{r}$ & load vector for the rotor finite-element model \\
$f_{s}$ & load vector for the pad finite-element model \\
\hline
\end{tabular}

${ }^{a}$ Corresponding author: acer@mek.dtu.dk

$\begin{array}{|ll|}E & \text { TPJB pad material elasticity modulus (Pa) } \\ E H D & \text { elastohydrodynamic lubrication regime } \\ E T H D & \text { elastothermohydrodynamic lubrication } \\ & \text { regime } \\ h & \text { oil film thickness (m) } \\ \boldsymbol{K}_{s} & \text { stiffness matrix of the pad finite-element } \\ & \text { model } \\ \boldsymbol{K}_{r} & \text { stiffness matrix of the rotor finite-element } \\ & \text { model } \\ \boldsymbol{K}_{g} & \text { stiffness matrix of the rotor-TPJBs model } \\ k_{c} & \text { oil thermal conductivity (W.m }{ }^{-1} \text {.K) } \\ k_{p} & \text { pad material thermal conductivity } \\ & \text { (W.m-1.K) } \\ L_{0} & \text { TPJB pad width (m) } \\ \boldsymbol{M}_{s} & \text { length of the oil injection hole (m) } \\ \boldsymbol{M}_{r} & \text { mass matrix of the rotor finite-element model } \\ \boldsymbol{M}_{g} & \text { mass matrix of the rotor-TPJBs model } \\ n_{0} & \text { number of injection holes on each pad } \\ n_{s} & \text { number of pads on the TPJB } \\ p & \text { oil film pressure (Pa) } \\ P_{\text {inj }} & \text { oil injection pressure (Pa) } \\ & \end{array}$




\begin{tabular}{|c|c|}
\hline$q_{g}$ & $\begin{array}{l}\text { vector degrees of freedom for the rotor-TPJBs } \\
\text { model }\end{array}$ \\
\hline$q_{r}$ & $\begin{array}{l}\text { vector degrees of freedom for the rotor finite- } \\
\text { element model }\end{array}$ \\
\hline$q_{s}$ & $\begin{array}{l}\text { vector degrees of freedom for the pad finite- } \\
\text { element model }\end{array}$ \\
\hline$q_{s}^{*}$ & $\begin{array}{l}\text { vector degrees of freedom for the reduced pad } \\
\text { finite-element model }\end{array}$ \\
\hline$R$ & rotor journal radius $(\mathrm{m})$ \\
\hline$R_{s}$ & TPJB pad inner radius (m) \\
\hline$T$ & oil temperature $\left({ }^{\circ} \mathrm{C}\right)$ \\
\hline$t$ & time $(\mathrm{s})$ \\
\hline THD & thermohydrodynamic lubrication regime \\
\hline$T P J B$ & tilting pad journal bearing \\
\hline$U$ & rotor journal tangential speed $\left(\mathrm{m} . \mathrm{s}^{-1}\right)$ \\
\hline$V_{\text {inj }}$ & injection velocity $\left(\mathrm{m} . \mathrm{s}^{-1}\right)$ \\
\hline$V_{s}$ & $\begin{array}{l}\text { pseudo modal matrix of the pad finite- } \\
\text { element model }\end{array}$ \\
\hline$x$ & global Cartesian coordinate system \\
\hline$y$ & global Cartesian coordinate system \\
\hline$z$ & global Cartesian coordinate system \\
\hline
\end{tabular}

\section{Introduction}

Among the oil film bearing designs, the tilting-pad journal bearing is widely used due to their superior stability properties. Hence, there has been a constant interest over the years to improve the knowledge regarding the characteristics of this mechanical element. Such research effort has been carried out by means of continuous improvement the available mathematical models, as well as by experimental investigation of its static and dynamic behavior. Within the modelling effort, a number of authors have made significant contributions to the development of this area. Starting with the work from Lund [1], and later Allaire [2], the ground was set for the calculation of dynamic coefficients for this type of bearings. From there on, a number of publications have dealt with the incorporation of more effects into the modelling of the tilting-pad journal bearing. Namely, flexibility effects, coming from pad and pivot deformation due to thermal growth and presssure loading, and thermal effects, due to the temperature build-up within the oil film, are nowadays included into the modelling of tiltingpad journal bearings. Hence, the state of the art within the modelling of these bearings corresponds to an elastothermo-hydrodynamic (ETHD) formulation, such as the ones presented in $[3,4]$. Together with the development of the knowledge within this subject, several strategies have been formulated in order to enhace the versatility of the tilting-pad journal bearing, transforming it into a smart machine element. One approach to do it corresponds to the one presented by Santos [5], which involves the injection of pressurized oil into the bearing clearance, through holes drilled across the pads. Since the injection pressure can be modified, a controllable lubrication regime is established, which can enable to modify the dynamic properties of the bearing in order to fit the operational requirements. Many publications have dealt with the application of this concept, such as [6-12].

This work is aimed at presenting an elasto-thermohydrodynamic (ETHD) model for the tilting-pad journal bearing with controllable lubrication. Such model is used to obtain theoretical results that show the feasibility of improving the thermal and dynamic properties of such bearing by means of the controllable lubrication scheme.

\section{Tilting-Pad Journal Bearing with controllable lubrication: mathematicals modelling}

Previous publications on the subject of controllable lubrication have established separately a Thermo-Hydrodynamic model (THD) and an Elasto-Hydrodynamic model $(E H D)$ for this lubrication regime. The main original contribution of this work correspond to the simultaneous implementation of both effects (ETHD) for the controllable lubrication Tilting-Pad Journal Bearing. For the sake of completeness, a brief presentation of such mathematical models is given here. The references provide a more complete presentation of such models.

\subsection{Modified Reynolds Equation}

The oil film pressure build up for the Tilting-Pad Journal Bearing has been traditionally described by means of the Reynolds Equation, based on the assumption of laminar flow and negligible effects of the fluid inertia and radial viscous shear forces. Such basic model was extended in [5], including some terms to model the effect of the oil injection into the bearing clearance. Hence, the Modified Reynolds Equation is established, as shown in Equation (1).

$$
\begin{gathered}
\frac{\partial}{\partial \bar{y}}\left(\frac{h^{3}}{\mu} \frac{\partial p}{\partial \bar{y}}\right)+\frac{\partial}{\partial \bar{z}}\left(\frac{h^{3}}{\mu} \frac{\partial p}{\partial \bar{z}}\right)-\frac{3}{\mu_{\mathrm{inj}} l_{0}} \sum_{i=1}^{n_{0}} F_{i}(\bar{y}, \bar{z}) \cdot p= \\
6 U \frac{\partial h}{\partial \bar{y}}-12 \frac{\partial h}{\partial t}-\frac{3}{\mu_{\mathrm{inj}} l_{0}} \sum_{i=1}^{n_{0}} F_{i}(\bar{y}, \bar{z}) \cdot P_{\mathrm{inj}}
\end{gathered}
$$

where $F_{i}(\bar{y}, \bar{z})$ is defined as follows:

$$
\begin{gathered}
F_{i}(\bar{y}, \bar{z})=\frac{d_{0}^{2}}{4}-\left(\bar{y}-\overline{y_{i}}\right)^{2}-\left(\bar{z}-\overline{z_{i}}\right)^{2}, \\
\text { if }\left(\bar{y}-\overline{y_{i}}\right)^{2}-\left(\bar{z}-\overline{z_{i}}\right)^{2} \leq \frac{d_{0}^{2}}{4} \\
F_{i}(\bar{y}, \bar{z})=0, \\
\text { if }\left(\bar{y}-\overline{y_{i}}\right)^{2}-\left(\bar{z}-\overline{z_{i}}\right)^{2} \geq \frac{d_{0}^{2}}{4}
\end{gathered}
$$

A number of publications [6-12] on the subject of actively lubricated tilting pad journal bearings base the modeling 
of the oil film under a controllable lubrication regime on Equation (1). The reader is advised to refer specially to [8] if a complete presentation of this mathematical model is required.

From Equation (1), it can be seen that for a controllable lubrication regime, the oil film pressure field $p(\bar{y}, \bar{z})$ is a function of the injection pressure $P_{\text {inj }}$ and the geometry of the injection hole (included into the $F_{i}(\bar{y}, \bar{z})$ function), as well as a function of the variables established by the traditional Reynolds Equation, namely rotor rotational speed $(U=\Omega \cdot R)$, oil viscosity $\mu$ and oil film thickness $h$. It can be seen that the function $F_{i}(\bar{y}, \bar{z})$ is related to the orifices position along the pad surface, given by the coordinates $\left(\bar{y}_{i}, \bar{z}_{i}\right)$, and the orifices diameter $d_{0}$. For a passive TPJB, $F_{i}(\bar{y}, \bar{z})$ is equal to zero, hence the term $P_{\text {inj }}$ corresponding to the oil injection pressure, vanishes. It can also be observed that the two dimensional domain for the study is defined along the axial and circumferential coordinates, in order to make room for the inclusion of the oil injection effects.

The extra terms in Equation (1) related to the oil injection are obtained as a result of the boundary conditions for the velocity field of the oil film. For a passive bearing, the oil velocity in the radial direction at the pad surface is set to be equal to the pad radial velocity, in order to respect the non-slip condition. For obtaining the Modified Reynolds Equation, one assumes that, on top of the movement of the pad, the oil velocity in the radial direction over an injection hole can be modelled using the velocity profile given by a fully developed laminar flow in a circular pipe. The integration of the continuity equation using such boundary condition entails the addition of the two extra terms to the standard Reynolds equation formulation, hence the Modified Reynolds Equation is obtained as shown in Equation (1).

\subsection{Energy equation}

The oil film temperature build up due to the shear and pressure forces developed within the fluid can be modelled by establishing an energy balance, which accounts for the variation of the fluid energy (kinematic and thermal) due to the work of the forces acting over it (namely pressure and shear forces). Furthermore, the effect of the oil injection into the clearance can be included as presented in $[13,14]$, obtaining the Energy Equation for Controllable Lubrication Regime, as given by Equation (3).

$$
\begin{aligned}
& \rho C_{p} h \frac{\partial T}{\partial t}+k_{c} h \frac{\partial^{2} T}{\partial \bar{y}^{2}}+k_{c} h \frac{\partial^{2} T}{\partial \bar{z}^{2}}+\left.k_{c} \frac{\partial T}{\partial \bar{x}}\right|_{0} F_{i} \\
& +\left(\frac{\rho C_{p} h^{3}}{12 \mu} \frac{\partial p}{\partial \bar{y}}-\frac{\rho C_{p} U h}{2}\right) \frac{\partial T}{\partial \bar{y}}+\frac{\rho C_{p} h^{3}}{12 \mu} \frac{\partial p}{\partial \bar{z}} \frac{\partial T}{\partial \bar{z}} \\
& \rho C_{p}\left(V_{\mathrm{inj}}-\frac{\partial h}{\partial t}\right)\left(T-T_{\mathrm{inj}}\right)=\frac{4}{3} \frac{\mu}{h}\left(V_{\mathrm{inj}}-\frac{\partial h}{\partial t}\right)^{2} \\
& p\left(V_{\mathrm{inj}}-\frac{\partial h}{\partial t}\right)-U^{2} \frac{\mu}{h}-\frac{h^{3}}{12 \mu}\left[\left(\frac{\partial p}{\partial \bar{y}}\right)^{2}+\left(\frac{\partial p}{\partial \bar{z}}\right)^{2}\right]
\end{aligned}
$$

The reader is advised to refer specially to [13] if a complete presentation of this mathematical model is required. The injection velocity profile $V_{\text {inj }}$ is determined as a completely developed laminar flow inside the injection orifice, using the expression given by Equation (4):

$$
V_{\mathrm{inj}}(\bar{y}, \bar{z})=-\frac{1}{4 \mu_{\mathrm{inj}} l_{0}}\left(P_{\mathrm{inj}}-p\right) \cdot \sum_{i=1}^{n_{0}} F_{i}(\bar{y}, \bar{z})
$$

Equation (3) enables to obtain the oil film temperature as a function of the oil film pressure field, bearing operational condition and oil thermal properties, as well as the injection parameters (namely, temperature and injection velocity, which is a function of the injection pressure). Physically, the extra terms corresponding to the effect of the oil injection account for the following effects: diffusive heat conduction between the oil film and the injection oil due to their temperature difference, convective heat transport of the injection oil when entering the bearing clearance, and work of the pressure and shear forces generated due to the high pressure oil injection.

It must be noted that the need of including the effect of the oil injection implies that no radial distribution for the oil film temperature is obtained, hence this formulation models the temperature field in the circumferential and axial direction, in the same way that the Modified Reynolds Equation defines the domain for the study. Secondly, the heat transfer effects between the oil film and the pad surface is included in a highly simplified way, by imposing the oil film temperature at the pad surface. Even though such boundary conditions equal to the existence of a non physical infinite heat transfer coefficient between fluid and solid, the results obtained using this model for bearings operating under laminar regime seems to be acceptable, see Section 4 of this paper. Thirdly, the boundary condition for this equation corresponds to the oil temperature at the leading edge of the pad, which is calculated by a simple mass and energy balance at the area between the pads (mixing zone).

Once the temperature field is calculated, it is possible to update the viscosity of the oil film by knowing the variation law of this parameter with the temperature, as given by Equation (5).

$$
\mu=\mu^{*} e^{-\beta\left(T-T^{*}\right)}
$$

where the $\mu^{*}$ and $\beta$ parameters are characteristics of the oil.

\subsection{Pad flexibility}

The inclusion of the pad elastic deformations due to the oil film pressure field is done by following a pseudo modal reduction scheme. This method was introduced in [3] and later implemented in $[10,11]$ in the context of the controllable lubrication regime. The reader is advised to refer to [10] in order to get further insights into this method. The basic idea consists of expressing the pad deformation as a linear combination of a finite number of 
the eigenmodes of the pads, calculated without including the presence of the oil film. The first eigenmode corresponds to the tilting motion of the pad around the pivot as a rigid body, whereas the higher modes correspond to pad elastic deformation shapes. Hence, the linear combination of such modes will generate a displacement of each point of the pad, where the elastic deformations and the tilting motion are included. Since the oil film thickness calculation is performed using that distorted shape, the solution of the Reynolds Equation and Energy Equation becomes a function of the pad flexibility effects.

In mathematical terms, by using the finite-element method the model of the pads can be expressed as:

$$
\boldsymbol{M}_{s} \ddot{\boldsymbol{q}}_{s}+\boldsymbol{K}_{s} \boldsymbol{q}_{s}=\boldsymbol{f}_{s}
$$

where $\boldsymbol{q}_{s}$ correspond to the degrees of freedom for each node of the finite element model, $\boldsymbol{M}_{s}$ and $\boldsymbol{K}_{s}$ correspond to the inertia and stiffness matrix for the pads, obtained using the finite-element method, and $\boldsymbol{f}_{s}$ represent the loads over the pads due to the pressure profile in the oil film. By calculating the pseudo-modal matrix $\boldsymbol{V}_{s}$ containing on its columns some of the eigenmodes of the pads, one can rearrange Equation (6) as follows:

$$
\begin{aligned}
\boldsymbol{V}_{s}^{T} \boldsymbol{M}_{s} \boldsymbol{V}_{s} \ddot{\boldsymbol{q}}_{s}^{*}+\boldsymbol{V}_{s}^{T} \boldsymbol{K}_{s} \boldsymbol{V}_{s} \boldsymbol{q}_{s}^{*} & =\boldsymbol{V}_{s}^{T} \boldsymbol{f}_{s} \\
\boldsymbol{q}_{s} & =\boldsymbol{V}_{s} \boldsymbol{q}_{s}^{*}
\end{aligned}
$$

By using the reduction scheme exposed in Equation (7), one ends working with a reduced system defined by the modal coordinates vector $\boldsymbol{q}_{s}^{*}$, where there are as many degrees of freedom as eigenmodes were included into the modal matrix $\boldsymbol{V}_{s}$. It corresponds to a pseudo-modal reduction, since only the eigenmodes which are relevant are included into the analysis. If only the first eigenmode is included, then a rigid pad model is established, and the corresponding modal coordinate measures the tilting of the pad around the pivot. The use of higher eigenmodes enables to include the flexibility of the pads into the results.

\subsection{Heat Conduction through the pads and Thermal Growth}

The heat conduction is modelled mathematically using the 3D form of the Fourier Heat Conduction Law, as given by Equation (8)

$$
k_{p} \frac{\partial^{2} T}{\partial x^{2}}+k_{p} \frac{\partial^{2} T}{\partial y^{2}}+k_{p} \frac{\partial^{2} T}{\partial z^{2}}=0
$$

Regarding the boundary conditions, the fluid film temperature is applied as boundary condition at the fluid-pad interface. The inlet mixing temperature is applied at the pad leading edge surface and the oil supply temperature is prescribed at the back of the pad. Again, the authors stress that such simple model equals to infinite heat transfer coefficients on the pad surfaces, which corresponds to a non physical behavior. However, considering the results shown in Section 4, it is kept due to its simplicity and obtained accuracy, which is enough for the scope of this study.

Once the temperature distribution is obtained for the pad, it is possible to calculate the thermal growth and its impact on the oil film thickness. To do so, a thermal expansion rule is applied to calculate the deformation related to a certain increment in the pad material temperature, as shown in Equation (9).

$$
\epsilon=\alpha \Delta T
$$

The thermal deformation as a result of the pad temperature field are calculated using Equation (9) and imposed to the pad finite-element model, on top of the pad pivoting motion and elastic deformations due to the pressure field loading. Regarding the shaft, its temperature is assumed to be non position dependant, and it is calculated as the average of the oil film temperature. Then, its thermal expansion is calculated using Equation (9).

\subsection{Numerical Implementation of the ETHD model}

The ETHD model developed on this work correspond to an extension of the one presented in $[10,11]$. In such work, the finite-element method was the method of choice for solving the partial differential equations corresponding to the Modified Reynolds Equation and Pad Flexibility Model using pseudo modal reduction scheme. Hence, the implementation of the oil film Energy Equation and Fourier Law for Heat Conduction on the Pads for this work is done using the finite-element method as well. The "solid" domain (pads) is discretized using tridimensional second order twenty node serendipity finite-elements. The "fluid" domain is discretized using bidimensional second order eight node quadrilateral elements, corresponding to one face of the "solid" serendipity elements. Hence, the link between the two domains is straightforward. The usage of second order elements is justified by the need of describing the pad geometry, specially the pad curvature and geometry of the injection orifice, in an accurate way.

The obtention of the weak form of the Modified Reynolds Equation and Fourier Law is done by using the Galerkin method. However, the usage of such method for the Energy equation induces numerical unstability on the solution, in the form of spurious oscillation on the obtained temperature values or "wiggle". This is a consequence of the inclusion of the oil injection terms in the Energy equation, which can be seen as the presence of a boundary condition in an upstream position, as well as the nature of the oil film flow, which exhibits a high Peclet number, in other words, strong dominance of convection effects over diffusion effects. To overcome this numerical unstability, the weak form of the Energy equation is obtained using a streamline upwind Petrov-Galerkin formulation, as presented in [15].

The dynamic behaviour of the Tilting-Pad Journal Bearing is linearized around its static equilibrium position by calculating the complete set of stiffness and damping coefficients using an analytical perturbation solution. 
Such method, introduced firstly in [1] and then extended in [2], enables to couple the bearing "local" behaviour with the rotor "global" dynamic behaviour in a compact and straightforward way.

\section{Rotor-Tilting Pad Journal Bearing system: mathematical modelling}

The rotor is modeled using the finite-element method, using shaft elements, as proposed by Nelson [16]. Proportional damping is included in order to model the shaft internal energy dissipation processes. Thus, a damping ratio of 0.001 is imposed on the first two flexible modes of the shaft. Hence, by using this method the inertia, stiffness, gyroscopic matrices and proportional damping matrices are obtained for the shaft.

By using this method, the system can be represented mathematically as follows:

$$
\boldsymbol{M}_{r} \ddot{\boldsymbol{q}}_{r}+\left(\boldsymbol{D}_{r}-\Omega \boldsymbol{G}_{r}\right) \dot{\boldsymbol{q}}_{r}+\boldsymbol{K}_{r} \boldsymbol{q}_{r}=\boldsymbol{f}_{r}
$$

In Equation (10), $\boldsymbol{q}_{r}$ represents the degrees of freedom of the nodes corresponding to the finite shaft model of the rotor, $\boldsymbol{M}_{r}$ is the rotor inertia matrix, $\boldsymbol{D}_{r}$ is the proportional damping matrix, $\boldsymbol{G}_{r}$ is the gyroscopic matrix and $\boldsymbol{K}_{r}$ is the rotor stiffness matrix. Related to the loading term $\boldsymbol{f}_{r}$, for this analysis the only loading applied to the system corresponds to the static load due to the weight of the rotor. The complete set of dynamic coefficients obtained for the Tilting-Pad Journal Bearings are assembled together with the shaft matrices, in order to obtain the global stiffness and damping matrices. By doing so, the model for the global system is defined as:

$$
\begin{array}{r}
\boldsymbol{M}_{g} \ddot{\boldsymbol{q}}_{g}+\left(\boldsymbol{D}_{g}-\Omega \boldsymbol{G}_{g}\right) \dot{\boldsymbol{q}}_{g}+\boldsymbol{K}_{g} \boldsymbol{q}_{g}=\boldsymbol{f}_{g} \\
\text { where } \boldsymbol{q}_{g}=\left\{\begin{array}{ll}
\boldsymbol{q}_{r} & \boldsymbol{q}_{s}^{*}
\end{array}\right\}^{T}
\end{array}
$$

The degrees of freedom for the global system $\boldsymbol{q}_{g}$ are defined by the rotor model degrees of freedom $\boldsymbol{q}_{r}$ and by the pads degrees of freedom $\boldsymbol{q}_{s}^{*}$, derived from the pseudo modal reduction.

The obtained global system is suitable to perform a number of analysis, such as: calculation of the Campbell diagram and stability map (eigenvalue calculation), unbalance response. To do so, the procedure to be followed, outlined in Figure 1 is depicted as follows:

1. Set the rotor rotational speed and the load applied over the bearing.

2. Determine the static equilibrium position of the rotorbearing system by means of a Newton Raphson Scheme. The Modified Reynolds Equation is solved to obtain the pressure field, which is integrated numerically to obtain resultant forces over pads and rotor. Convergence below a given tolerance value is to be achieved for the load equilibrium over the rotor and bearing pads. Once convergence is achieved, the system state is defined by the rotor position inside the bearing, as well as a number of modal coordinates, representing the tilting angle and elastic deformation of each pad corresponding to the modes included in the analysis.

3. Calculate the temperature field using the Energy equation. Since mass and energy balance calculation is performed in the mixing zone between two adyacent pads in order to obtain the oil film leading edge temperature, it is necessary to iterate to convergence. Once convergence on the temperature field is achieved, the oil film viscosity is updated, as well as the thermal growth of the pads and shaft.

4. Repeat 2 and 3 until convergence on the oil film viscosity is achieved. Such condition implies that the system has achieved steady state regime, hence both load and thermal equilibrium is established.

5. Using the perturbation solution, determine the complete set of dynamic coefficients for each bearing.

6. Assemble the global system matrices, including the inertia, stiffness, damping and gyroscopic matrix from the rotor finite-element model, and the complete set of dynamic coefficients of the tilting-pad journal bearings.

7. Using the global system matrices, perform an eigenvalue calculation for obtaining the Campbell diagram or the stability map, or use a synchronous vibration assumption to obtain the steady state unbalance response.

\section{Validation of the ETHD model for the Tilting-Pad Journal Bearing}

The obtained ETHD code for the Tilting-pad Journal Bearing is validated against theoretical and experimental data coming from the literature. Among the variables to be studied are: static equilibrium position in the form of oil film thickness, oil film pressure, temperature on the surface of the pad for the steady state regime and synchronously reduced dynamic coefficients. The validation of the ETHD model for the controllable lubrication is being undertaken now by the authors of this work, by experimental means. Some experimental results proving the validity of the controllable lubrication model can be found in [9].

\subsection{Validation against Fillon et al. [4,17]}

In [4], an ETHD model for the tilting-pad journal bearing is presented. An extensive experimental and theoretical study on the thermal behaviour of tilting-pad journal bearings is given in [17]. Such theoretical and experimental results are used to benchmark our code. They correspond to: oil film thickness and pressure field for the static equilibrium position, as well as temperature on the surface of the pads for the studied bearing. The comparison between those benchmark results and the ones obtained using our code are shown in (Fig. 2).

In general, close agreement is obtained between the benchmark results and the ones obtained using our ETHD 


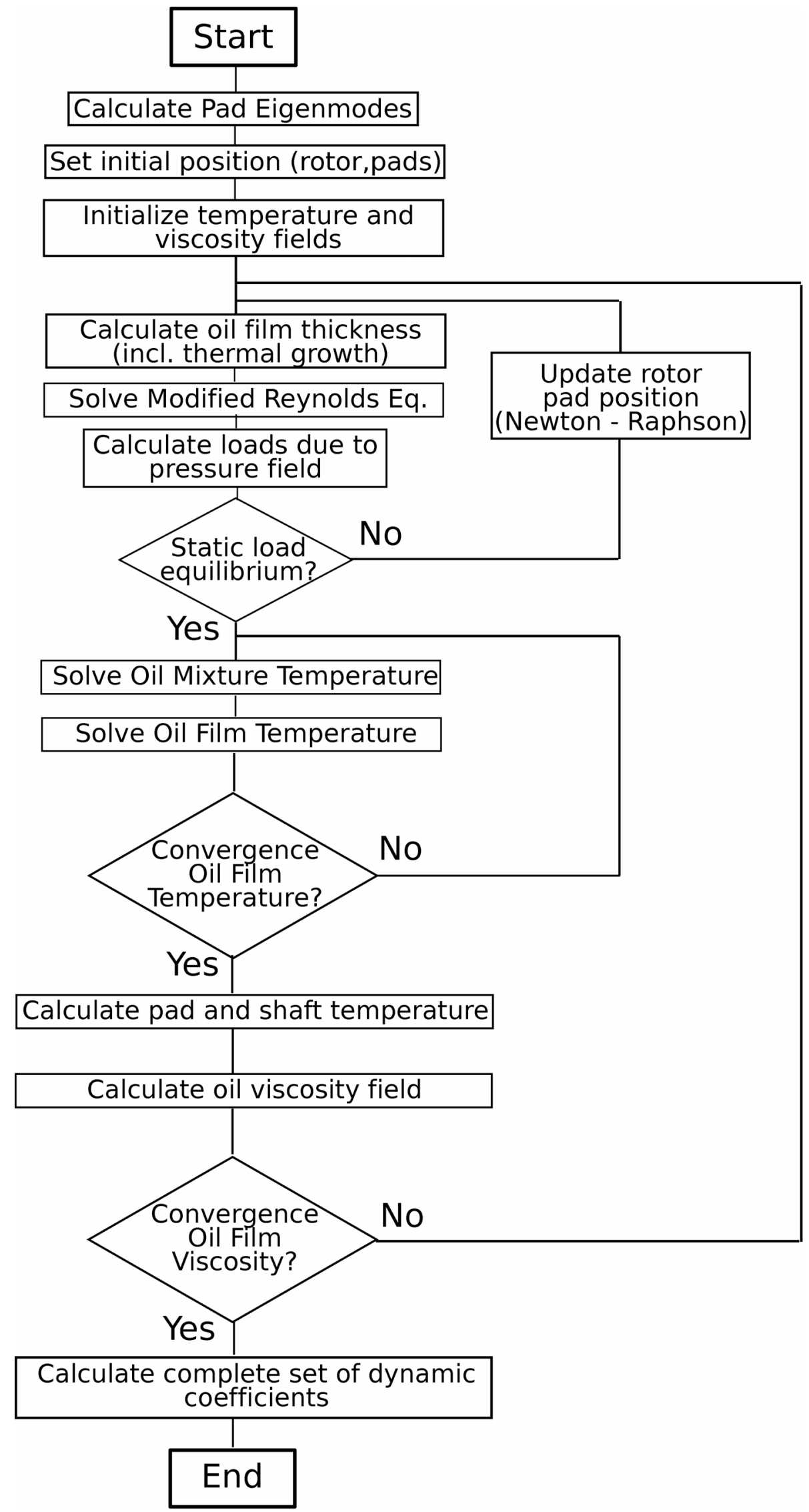

Fig. 1. Flow diagram for the model of the tilting pad journal bearing with controllable lubrication. 

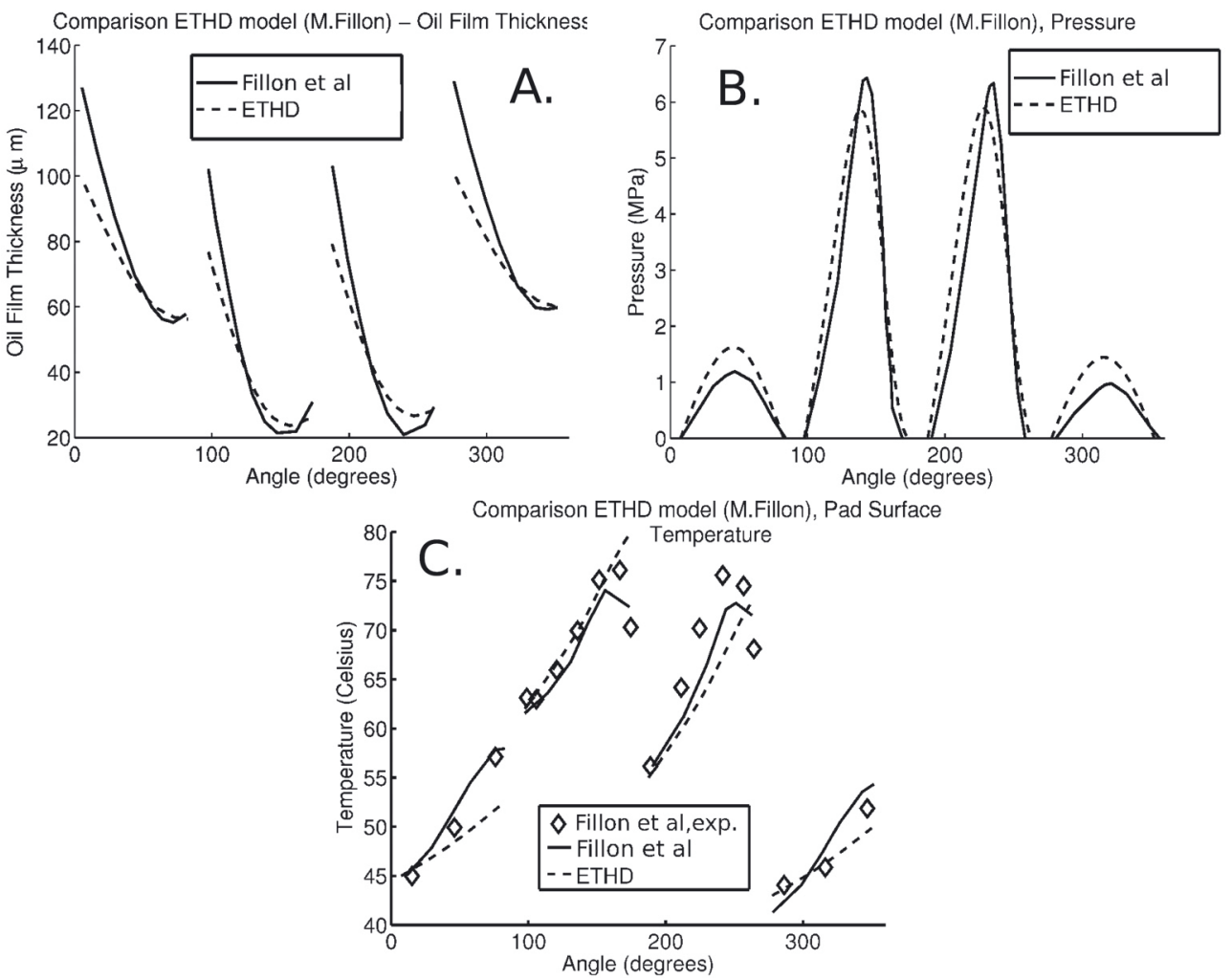

Fig. 2. Comparison between ETHD code and results from Fillon et al. [4,17]; the results shown correspond to oil film thickness (A), pressure profile (B) and pad surface temperature (C) at the static equilibrium position, for bearing A, as given in [17].

code. Even tough an simplified heat transfer model is assumed in our code, the results fits quite well with the provided experimental temperature data, (Fig. 2C), except for the zone towards the trailing edge. In such zone, both the experimental and theoretical benchmark results exhibit a drop in the pad surface temperature values, due to the heat transfer process taking place between the pad trailing edge and the oil bath. Since our model takes into account the heat transfer between pad and surroundings in a simplified way, it fails to reproduce the measured temperature drop.

\subsection{Validation against Taniguchi et al. [18]}

In [18], the authors present an analytical model and experimental results regarding the steady state pressure profile, pad surface temperature and oil film thickness for a large tilting pad journal bearing, operating in turbulent regime. Even tough such regime escapes the assumptions in which our code is based, this set of experimental data has been used by other authors, such as $[17,19]$, to validate their "laminar" codes. Our comparison against those experimental results is given in (Fig. 3).

Close agreement is achieved between the results from our code and the theoretical and experimental results reported in [18], regarding pressure profile and oil film thickness. From the temperature results, (Fig. 3C), it is clear that the assumption of pad surface temperature to be equal to oil film temperature is not valid when a turbulent regime is reached, as it leads to an underestimation of the pad surface temperature. However, the developed code is not meant to be used for bearings operating in such condition, since no provision for turbulent regime has been implemented, so this shortcoming corresponds to an expected result. Moreover, the results obtained for oil film thickness and pressure profile indicate that the static equilibrium position is being calculated accurately.

\subsection{Validation against Brockwell et al. [20]}

In [20], results regarding the synchronously reduced dynamic coefficients obtained for a five shoe tilting pad journal bearing are presented. Such results are obtained both by experimental and theoretical means, using an ETHD code. This set of results is used to benchmark the code developed in [3]. The comparison between the results obtained using our code and the ones used as benchmark is given in (Fig. 4).

In general terms, good agreement is obtained between our results and the ones given in [20]. Closer agreement is obtained for the stiffness coefficients than for the damping ones, in a similar way to the results presented in [3], when performing the same comparison. It can also be noted that the results from our code tend to overstimate the damping characteristics of the bearing, as expected since 

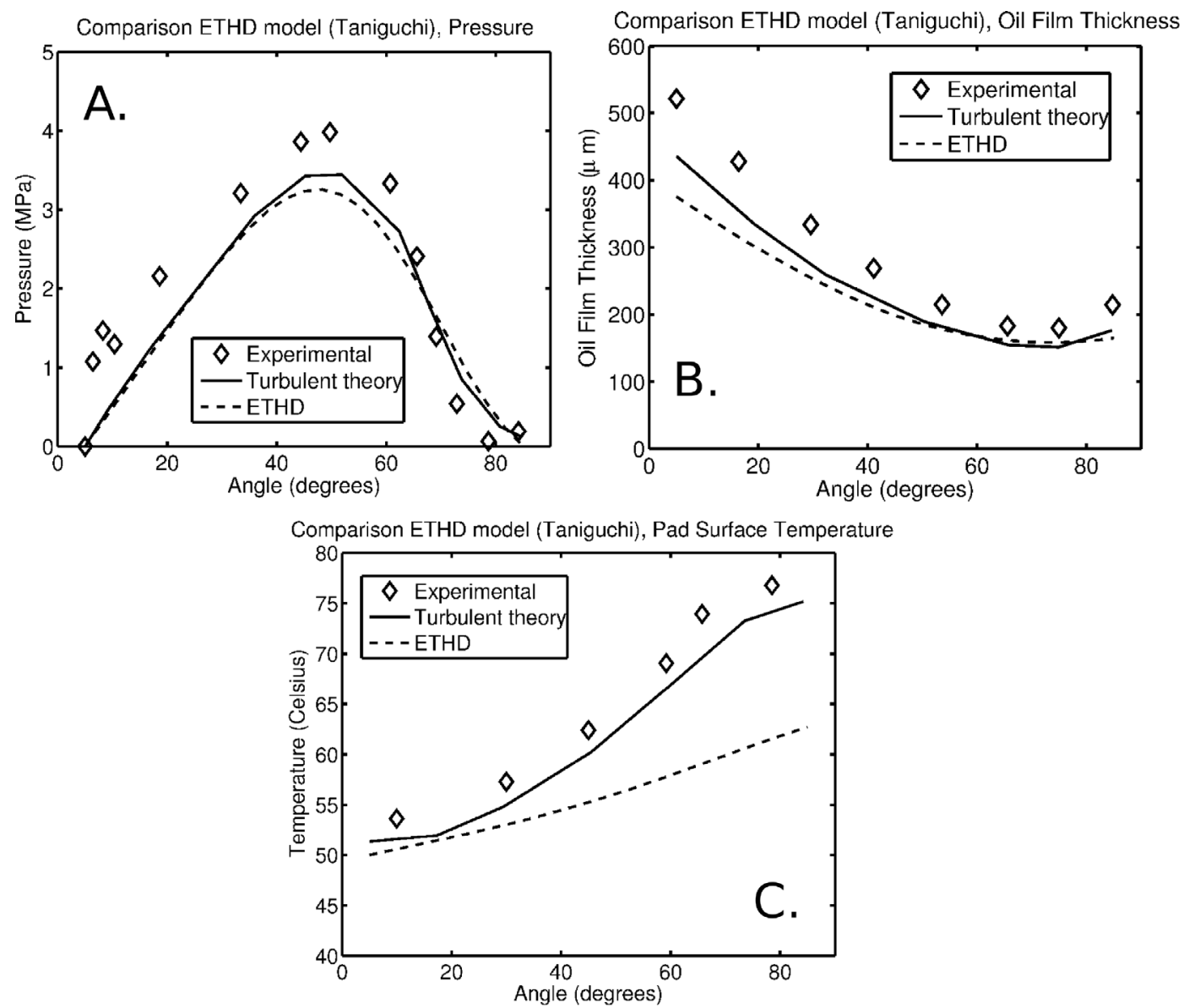

Fig. 3. Comparison between ETHD code and results from Taniguchi et al. [18]; pressure profile (A), oil film thickness (B) and pad surface temperature (C) for pad number 2, as given in [18].

pivot flexibility effects are not being included into the model.

\section{Analysis of an industrial compressor mounted over tilting-pad journal bearings with controllable lubrication}

The ETHD model for the tilting-pad journal bearing operating in a controllable lubrication regime is applied now to analyze an industrial rotor system. The objective is to determine the feasibility of improving the thermal behaviour of the bearing and the dynamic behaviour of a rotor mounted on such mechanical elements when pressurized oil is injected directly into the bearing clearance. These results correspond to an extension of the ones presented in [12], since those were obtained using an isothermal rigid-pads modeling assumption.

\subsection{Analyzed system and scope of the analysis}

The analyzed system is represented in (Fig. 5). The rotor corresponds to a gas compressor, composed of five impellers. It weighs $391 \mathrm{~kg}$, and it operates normally within the range of $6942 \mathrm{rpm}$ and $10170 \mathrm{rpm}$. The rotor is supported by two identical tilting pad journal bearings. The loading due to the rotor weight acts on the negative y direction, hence pad 4 becomes the most heavily loaded one. The impellers, seals and other machine elements are considered as rigid discs and are incorporated into the model by adding inertia to the respective nodes. Hence, in the model, the impellers are at nodes 20, 24, 28, 32 and 36 . Bushings are at nodes 22, 26, 30, and 34. A thrust disk sleeve is located at node 3 . A balance piston is located at node 38. Seal bushes are located at nodes 12 and 46 . Coupling is at node 55 .

As for the TPJBs, they are located at nodes 8 (bearing 1) and 50 (bearing 2). An injection hole is located on each pad, on an axially centered position, towards the leading edge. Table 1 depicts all the parameters that define the geometry of such bearings, as well as the oil properties.

The discretization of the shaft, using beam elements, and of the pads, using tridimensional second order twenty node serendipity finite-elements, is also shown in (Fig. 5). The elements used for the pad discretization are distributed as follows: 4 elements in the radial direction, 16 elements in the axial direction and 48 elements in the circumferential direction. Only half of the pad in the axial 

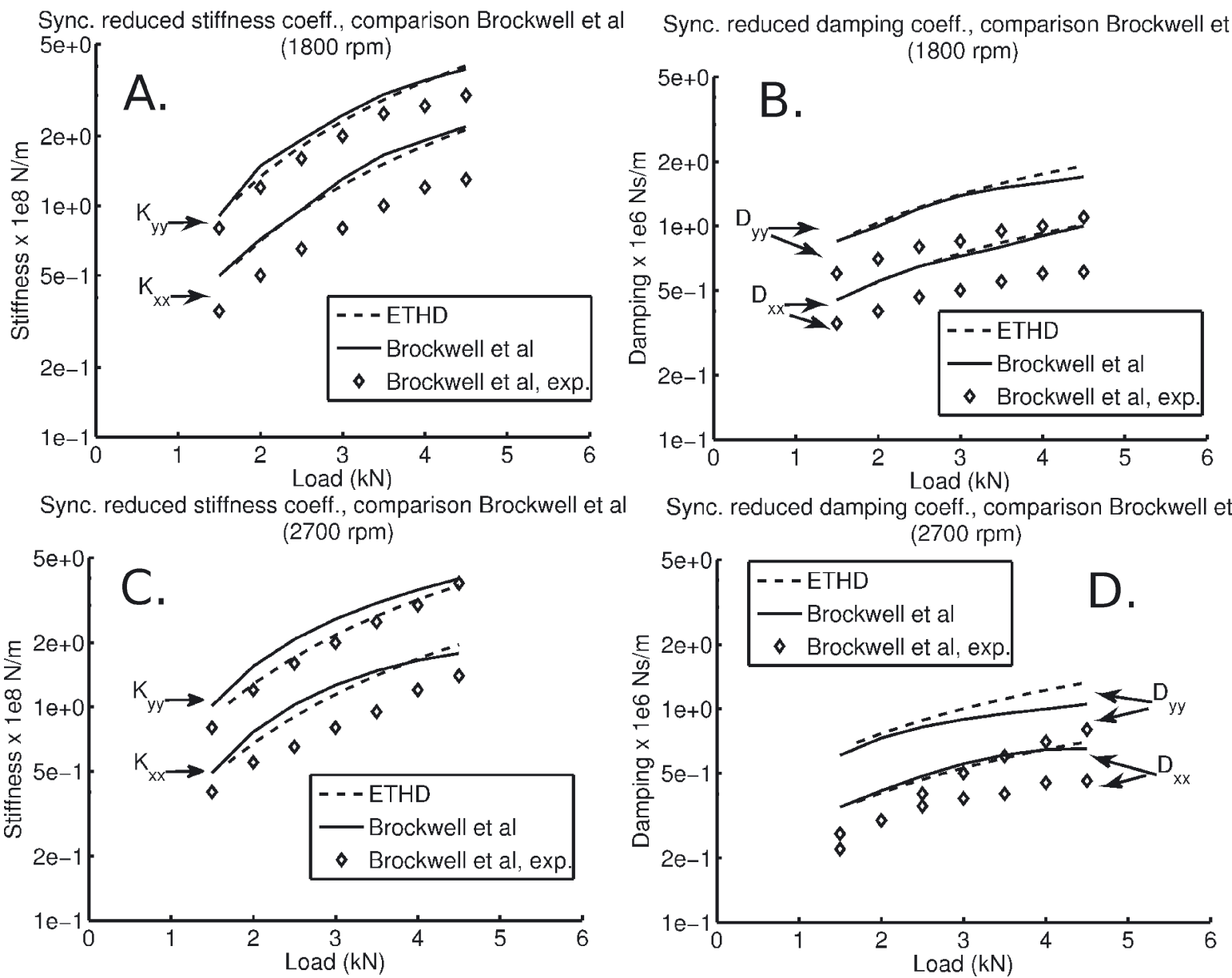

Fig. 4. Comparison between ETHD code and results from Brockwell et al. [20]; synchronously reduced dynamic coefficients obtained analytically and experimentally for a five shoe tilting-pad journal bearing, for two different rotational speeds.
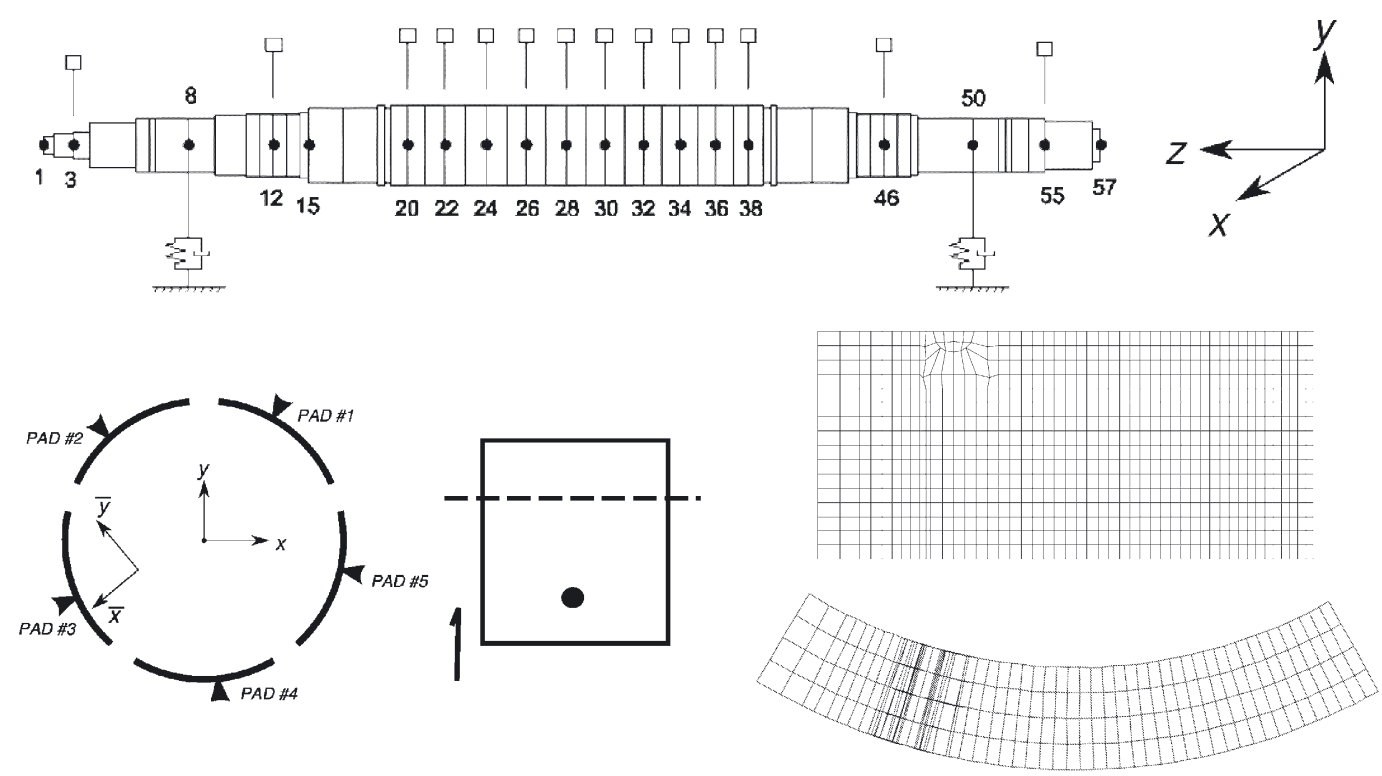

Fig. 5. Industrial compressor modeled using the finite-element method, mounted over two identical tilting-pad journal bearings. The finite-element discretization for the shaft and the pads is shown. The position of the injection hole on each pad with respect to the pivot line and leading edge is also depicted. 
Table 1. Analyzed system parameters.

\begin{tabular}{ccc}
\hline Parameter & Value & Units \\
\hline Journal Radius $(R)$ & 50.800 & $\mathrm{~mm}$ \\
Number of pads $\left(n_{s}\right)$ & 5 & - \\
Pad inner Radius $\left(R_{s}\right)$ & 50.921 & $\mathrm{~mm}$ \\
Pad aperture angle $\left(\alpha_{0}\right)$ & 60 & $\mathrm{deg}$ \\
Angular position pivot pad \#1 & 60 & $\mathrm{deg}$ \\
Angular position pivot pad \#2 & 132 & $\mathrm{deg}$ \\
Angular position pivot pad \#3 & 204 & $\mathrm{deg}$ \\
Angular position pivot pad \#4 & 276 & $\mathrm{deg}$ \\
Angular position pivot pad \#5 & 348 & $\mathrm{deg}$ \\
Offset & 0.6 & - \\
Pad width $(L)$ & 44.450 & $\mathrm{~mm}$ \\
Pad thickness $(\Delta s)$ & 10.312 & $\mathrm{~mm}$ \\
Pad material elasticity modulus $(E)$ & 200 & $\mathrm{GPa}$ \\
Pad material Poisson ratio $(\nu)$ & 0.3 & - \\
Pad material density $\left(\rho_{s}\right)$ & 7800 & $\mathrm{~kg} \cdot \mathrm{m}{ }^{-3}$ \\
Injection orifice length $\left(l_{0}\right)$ & 5.0 & $\mathrm{~mm}$ \\
Injection orifice diameter $\left(d_{0}\right)$ & 5.0 & $\mathrm{~mm}$ \\
Number of orifices per pad $\left(n_{0}\right)$ & 1,2 & - \\
Assembled bearing gap $\left(h_{0}\right)$ & 102 & $\mu \mathrm{m}$ \\
Oil viscosity $\left(40{ }^{\circ} \mathrm{C}\right)(\mu)$ & 0.028 & $\mathrm{~Pa} \mathrm{~s}$ \\
Oil viscosity $\left(80{ }^{\circ} \mathrm{C}\right)(\mu)$ & 0.007 & $\mathrm{~Pa} \mathrm{~s}$ \\
Oil density $(\rho)$ & 863.5 & $\mathrm{~kg} . \mathrm{m}^{-3}$ \\
Oil specific heat $\left(C_{p}\right)$ & 1900 & $\mathrm{~J} \cdot \mathrm{kg}^{-1} \cdot \mathrm{K}$ \\
Oil thermal conductivity $\left(k_{c}\right)$ & 0.13 & $\mathrm{~W} \cdot \mathrm{m}^{-1} . \mathrm{K}$ \\
Oil supply temperature & 50 & ${ }^{\circ} \mathrm{C}$ \\
Oil supply flow rate & 0.000375 & $\mathrm{~m}{ }^{3} \cdot \mathrm{s}^{-1}$ \\
Unbalance for the third impeller & 720 & $\mathrm{~g} \cdot \mathrm{mm}$ \\
Unbalance for the rotor coupling & 360 & $\mathrm{~g} \cdot \mathrm{mm}$ \\
\hline
\end{tabular}

direction is modeled, in order to take advantage of the axial symmetry of the pad geometry. Regarding convergence behavior for the pad model, the number of elements employed for the modeling is higher than the one used for modeling the bearings when comparing against the experimental results from the literature, as exposed in Section 4. On top of that, the guidelines obtained from the convergence analysis for the bearing model performed in [21] are taken into account.

The analysis is performed following the procedure stated in Section 3, using the validated ETHD code. In total, ten modes are included for the pseudo modal reduction scheme, five of them correspond to the tilting motion of each pad, and the remaining five correspond to the first bending mode of each pad. According to the results from [12], the inclusion of higher bending modes for the pads does not affect significantly the results concerning static equilibrium position and global system dynamic behavior. The results obtained allow to compare the behavior of the passive system (no injection holes are present in the bearings) against the controllable system, operating with two different injection pressures. Among the analysis performed, the oil film pressure and temperature field are compared for the two systems. Also, some tribological magnitudes are studied, such as minimum oil film thickness and oil temperature, as well as the dynamic behavior of the rotor-bearing systems, in the form of unbalance response and stability map.

\subsection{Oil film pressure and temperature field}

A comparison for the oil film pressure and temperature field for the passive system (no injection) and the controllable one is given in (Figs. 6 and 7). The results correspond to the static and thermal equilibrium position of bearing 2, operating at $4500 \mathrm{rpm}$ and $9000 \mathrm{rpm}$. None of the pads of the bearings was cavitating for the operational conditions simulated, although the model is capable of accounting for it using Gumbel model. Two different injection pressures are applied (20 bar and 40 bar).

It can be seen that by injecting pressurized oil the pressure and the temperature field are modified. A high pressure area is created towards the leading edge of the pad, around the injection hole. When analyzing the temperature field, it can be seen that the effect of the injected oil is to generate a stream of cooler oil. The flow area affected by this stream depends on the oil film pressure gradients and injection pressure, being larger for the lightly loaded pads and for higher injection pressure.

It can also be seen that the flow area unperturbed by the stream of cooler oil develops a higher temperature towards the trailing edge, when compared to the no injection case. This is particularly evident for (Fig. 7F); in this picture, it can be seen that a high temperature zone is created toward the corners of the trailing edge. According to the analysis performed in [12], the oil injection in the leading edge induces an increase of the tilting angle of the pad. Such increase will induce a reduction of the minimum oil film thickness towards the trailing edge, hence when the oil is forced to pass through that area, it develops a higher temperature, when compared to the "no injection" case.

\subsection{Minimum oil film thickness and oil temperature}

As it was mentioned in Section 5.2, by injecting pressurized oil towards the leading edge, an increase on the tilting angle of the pad is obtained, which entails a reduction in the minimum oil film thickness. The results shown in (Fig. 8A) are consistent with such analysis. Also, the increase on the oil film maximum temperature with injection pressure shown in (Fig. 8B) is consistent with the analysis exposed in Section 5.2. However, when analyzing the oil film average temperature results (Fig. 8C), a clear benefit from using the controllable lubrication regime arises, since a clear reduction is obtained for the analyzed rotational speeds.

At this point, it is worth mentioning that the local increase of oil temperature towards the trailing edge can also be a consequence of the adiabatic assumption for the oil film behavior. In Section 4.1, it was noted that our model fails to reproduce the oil temperature drop towards the trailing edge observed in the experimental data. Hence, it can be argued that the negative results obtained for the oil film maximum temperature when using the controllable lubrication can be a consequence of the simplifications introduced into the model, namely the lack of the modelling of the heat transfer effects between 

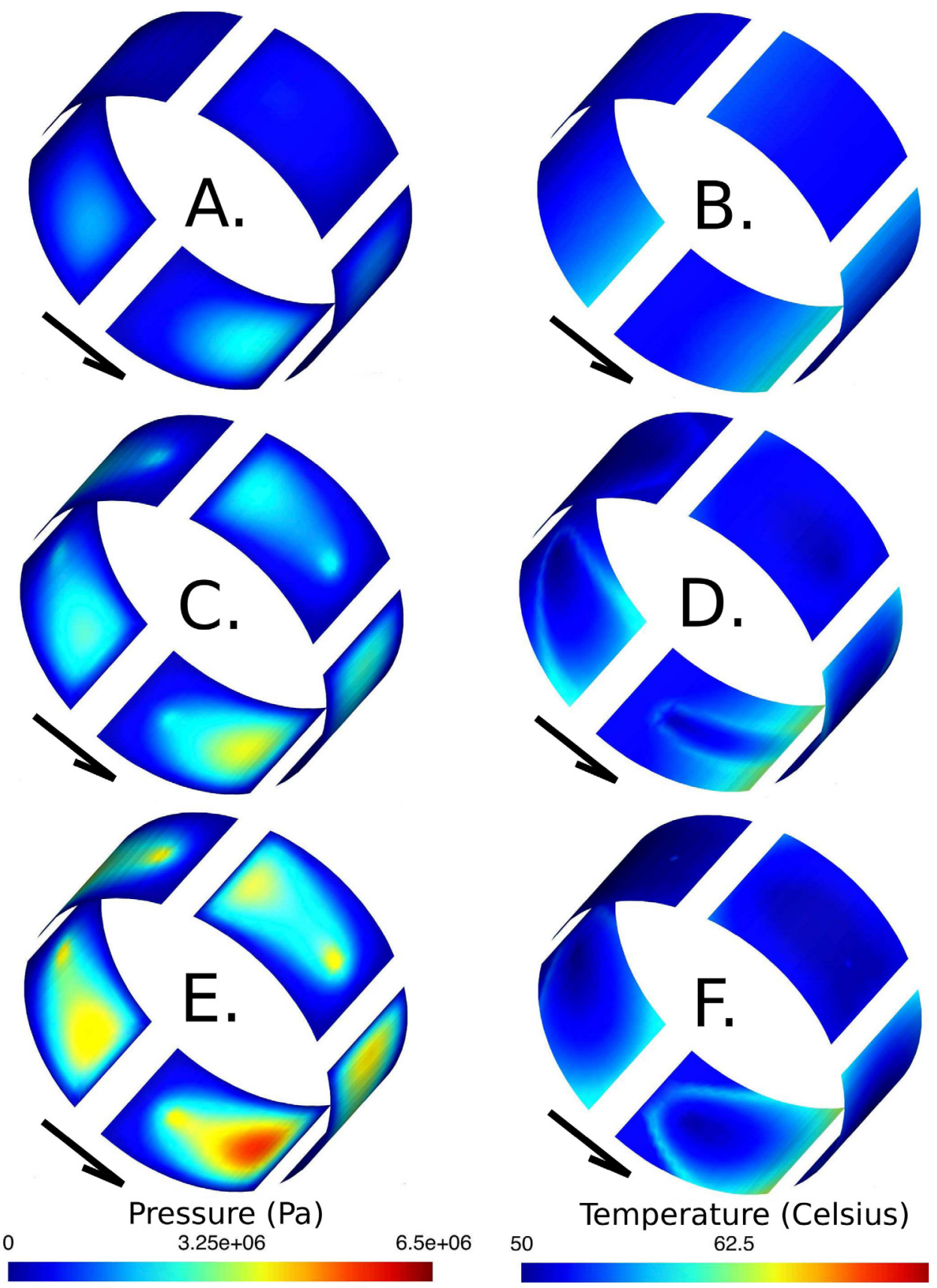

Fig. 6. Oil film pressure (A, C, E) and temperature field (B, D, F), in bearing 2, for $4500 \mathrm{rpm}$; comparison for no injection (A, $\mathrm{B}), 20$ bar injection pressure (C, D), 40 bar injection pressure (E, F).

oil film and bearing pads. Experimental testing is planned to prove the validity of this hypothesis.

\subsection{Dynamic behavior: rotor unbalance response and stability diagram}

By imposing a controllable lubrication regime, it is possible to modify the equilibrium position of the rotorbearing system, which entails a modification of the tiltingpad journal bearing dynamic properties. Both its stiffness and damping characteristics are modified, according to the theoretical results presented in [12]. Hence, it could be feasible to improve the performance of the rotor bearing system from the dynamic point of view, by a proper positioning of the injection orifices and proper selection of the injection pressure.

In (Fig. 9), the unbalance response amplitude for the rotor system, measured at node 28 , is shown. To obtain these results, an unbalance force was applied firstly to the rotor node 28, (Figs. 9A and B), which corresponds to the position of the third impeller of the compressor, and to node 55, (Figs. 9C and D), which corresponds to the 

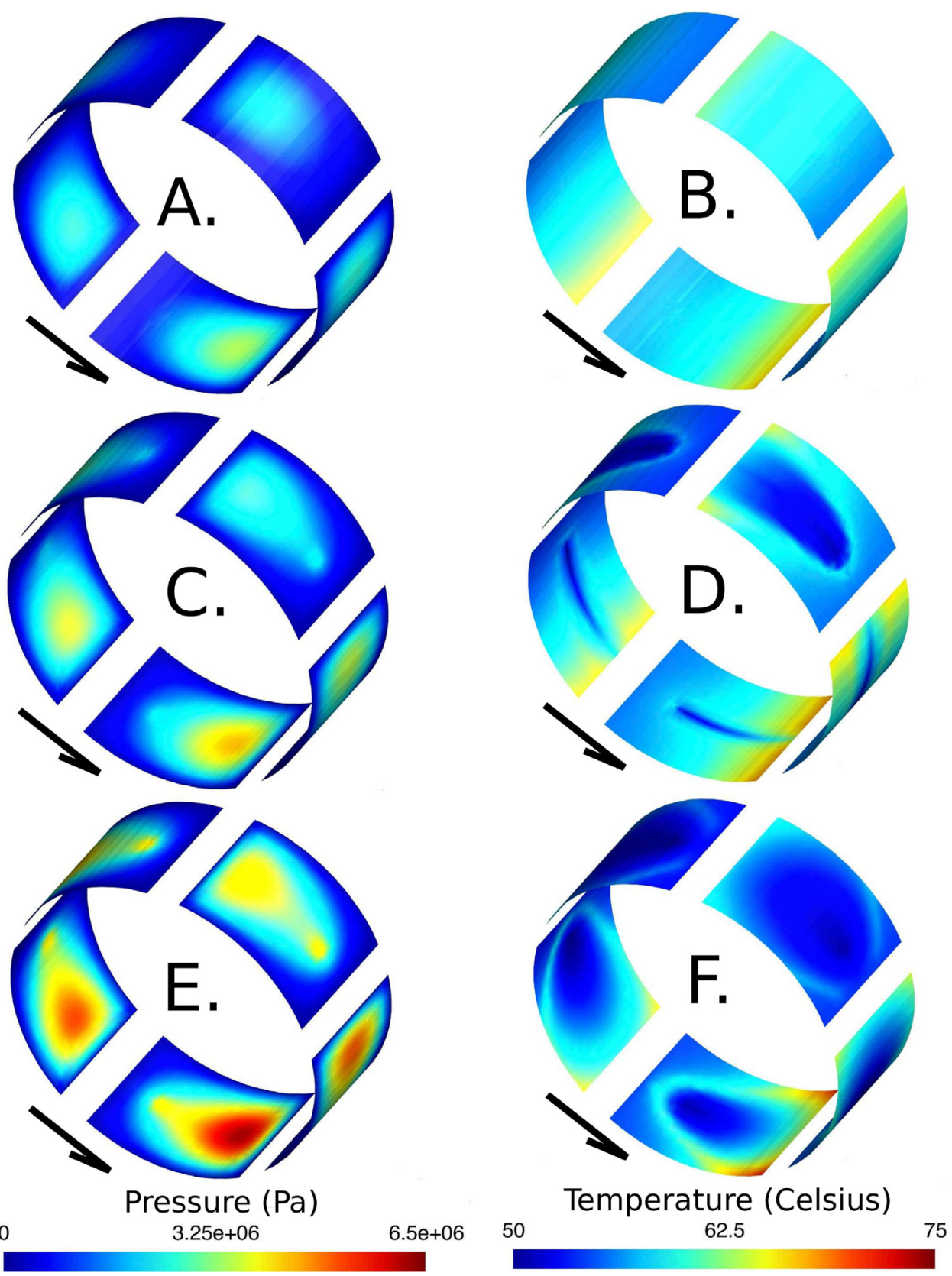

Fig. 7. Oil film pressure (A, C, E) and temperature field (B, D, F), in bearing 2, for $9000 \mathrm{rpm}$; comparison for no injection (A, B), 20 bar injection pressure (C, D), 40 bar injection pressure (E, F).

coupling. The value for the unbalance is $720 \mathrm{~g} . \mathrm{mm}$ for the third impeller, and 360 g.mm for the coupling, according to API 617. In (Figs. 9A and B), it can be observed that when injecting pressurized oil the position of the resonant peak is shifted to the left, which implies a reduction in the stiffness characteristics of the bearings. This analysis is also confirmed by the amplitude response to the left of the resonant peak, which becomes higher for the controllable lubrication cases. Such reduction in the stiffness characteristics is obtained alongside an increase in the damping ratio associated to the unbalance response of the rotor, responsible for the reduction in the amplitude of the resonant peak for the controllable case. Hence, these results show that the injection of oil towards the leading edge modify the stiffness and damping characteristics of the bearing, resulting in an increase of the resulting damping ratio for the rotor-bearings system. When comparing with the admissible vibration amplitude for the rotor according to API 617, the results show that by imposing the controllable lubrication regime with injection pressure of 20 bar, it is possible to reduce the unbalance response in order to comply with the requirement of the norm.

The results obtained for the stability map shown in (Fig. 10) support the previous results. A clear increase of the instability onset speed is obtained for the controllable case, due to an increment of the damping ratio associated 

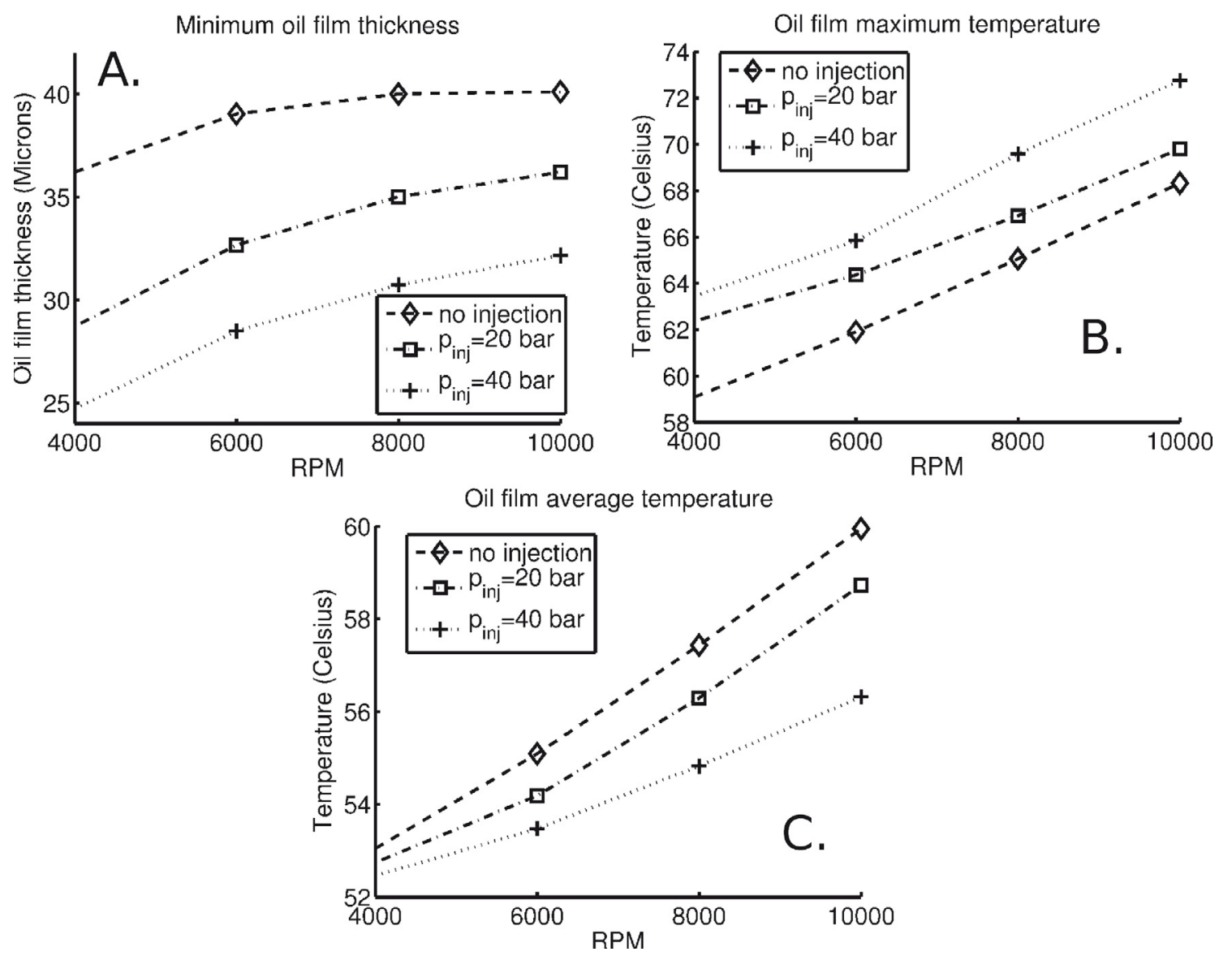

Fig. 8. Minimum oil film thickness (A), maximum (B) and average oil temperature (C) for bearing 2 operating at different rotational speeds; comparison for no injection, 20 bar and 40 bar injection case.

with the vibration mode approaching unstability, hence expanding the stable operational range of the compressor. These results confirm the ones obtained in [12], analysis that was performed using a rigid-pad isothermal model for the tilting-pad journal bearings.

With regards to the effect of changing the value of the injection pressure, the results from (Figs. 9A and B) and (Fig. 10) show opposite trends. For the unbalance response, when exciting at node 28 , an increase in the injection pressure entails a reduction in the damping ratio, whereas for the stability map, an increase in the injection pressure enlarges the stable operational range, implying higher damping ratio. This apparent contradiction can be explained, since the eigenmode being excited in the unbalance response plot (excitation at the impeller) is different than the one approaching the unstability threshold in the stability map. Since each one of these two different modes associated with different modal damping ratios, it can be inferred that a change in the injection pressure value modifies in different ways the damping ratio associated with different modes.

This analysis is supported by the results shown in (Fig. 11). The damping ratio for two different modes of the rotor bearing system is calculated for the analyzed range of rotational speed. Mode 1 corresponds to the eigenmode approaching unstable behavior, as noted in
(Fig. 10), whereas mode 2 is the mode excited due to the unbalance force in (Fig. 9). Firstly, the difference in the order of magnitude between the damping ratios for each mode must be noted. For both modes, it can be seen that the use of a controllable lubrication regime implies an increment in the damping ratio associated with the mode. However, for mode 1, an increase in the injection pressure value entails higher modal damping ratio, whereas for mode 2 the modal damping ratio is diminished for an increase in the injection pressure.

The analysis performed so far is valid for the unbalance response obtained when the excitation force is located at node 28, namely the third impeller, as shown in (Figs. 9A and B). The position of such node implies that the eigenmodes already shown in (Fig. 11) are preferably excited. However, when locating the unbalance force at the coupling of the rotor (node 55), namely at the end of the rotor, higher eigenmodes are excited, hence the response of the system becomes the result of the superposition of different modes. Therefore, the results shown in (Fig. 9) illustrate that the relationship between response amplitude and injection pressures seems to be altered,(Figs. 9C and D), when comparing with the case where the excitation was located at one of the impellers, (Fig. 9A and B). When exciting at the coupling, the amplitude of the resonant peak almost does not vary for the 

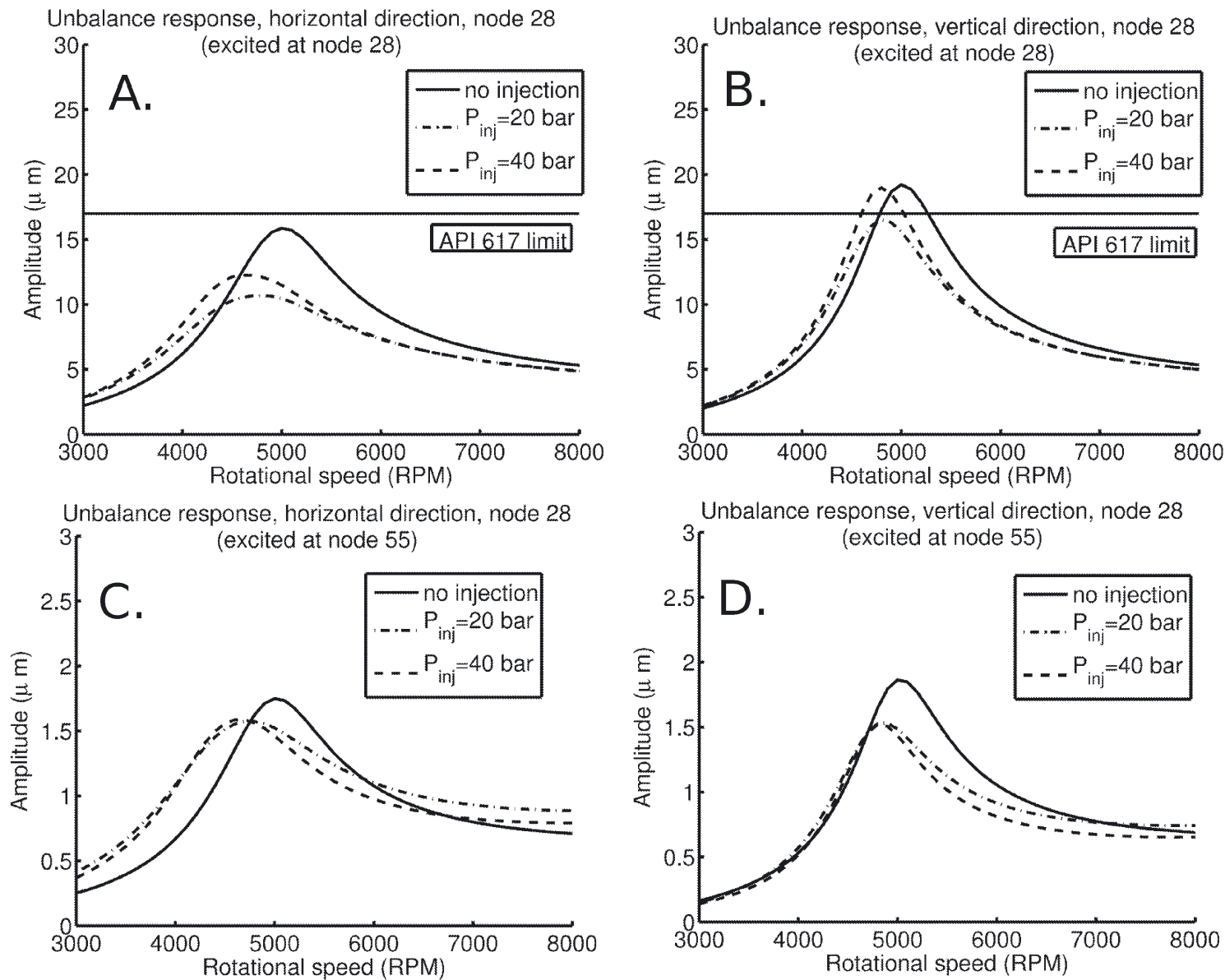

Fig. 9. Maximum amplitude for the unbalance response, measured at rotor node 28 (third impeller), for two different positions of the unbalance excitation (node 28, third impeller (A, B); node 55, coupling (C, D)); comparison for the passive case and controllable case, with different injection pressures.

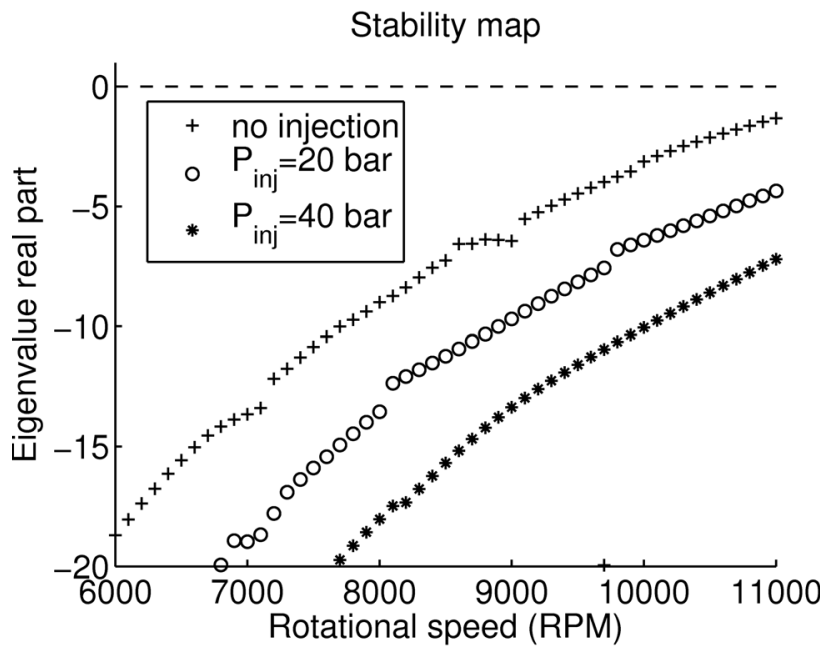

Fig. 10. Stability map of the compressor; the instability onset zone is shown for the no injection case and two different injection pressures.

imposed values of injection pressure, whereas the response outside the resonant peak does not converge towards the same value. These results show the difficulties associated with finding an optimum value for a fixed injection pressure on a practical application of the controllable (hybrid) lubrication scheme. Hence, the advantages of determining the value for the injection pressure by means of a control loop (active lubrication) become evident. Even tough in this work it has been theoretically showed that by injecting oil at a fixed pressure towards the leading edge of each pad it is possible to modify favourably the dynamic behavior of the rotor system, the achievement of the optimal results regarding reduction of the unbalance response requires varying the injection pressure according to the system state, by means of a control loop, as it was shown theoretically and experimentally in other works on these subject [9].

\section{Conclusion}

In this work, an Elasto-Thermo-Hydrodynamic (ETHD) model for the Tilting-Pad Journal Bearing has been presented. Such model includes also the effect of injecting high pressure oil directly into the bearing clearance through orifices across the bearing pads, allow- 

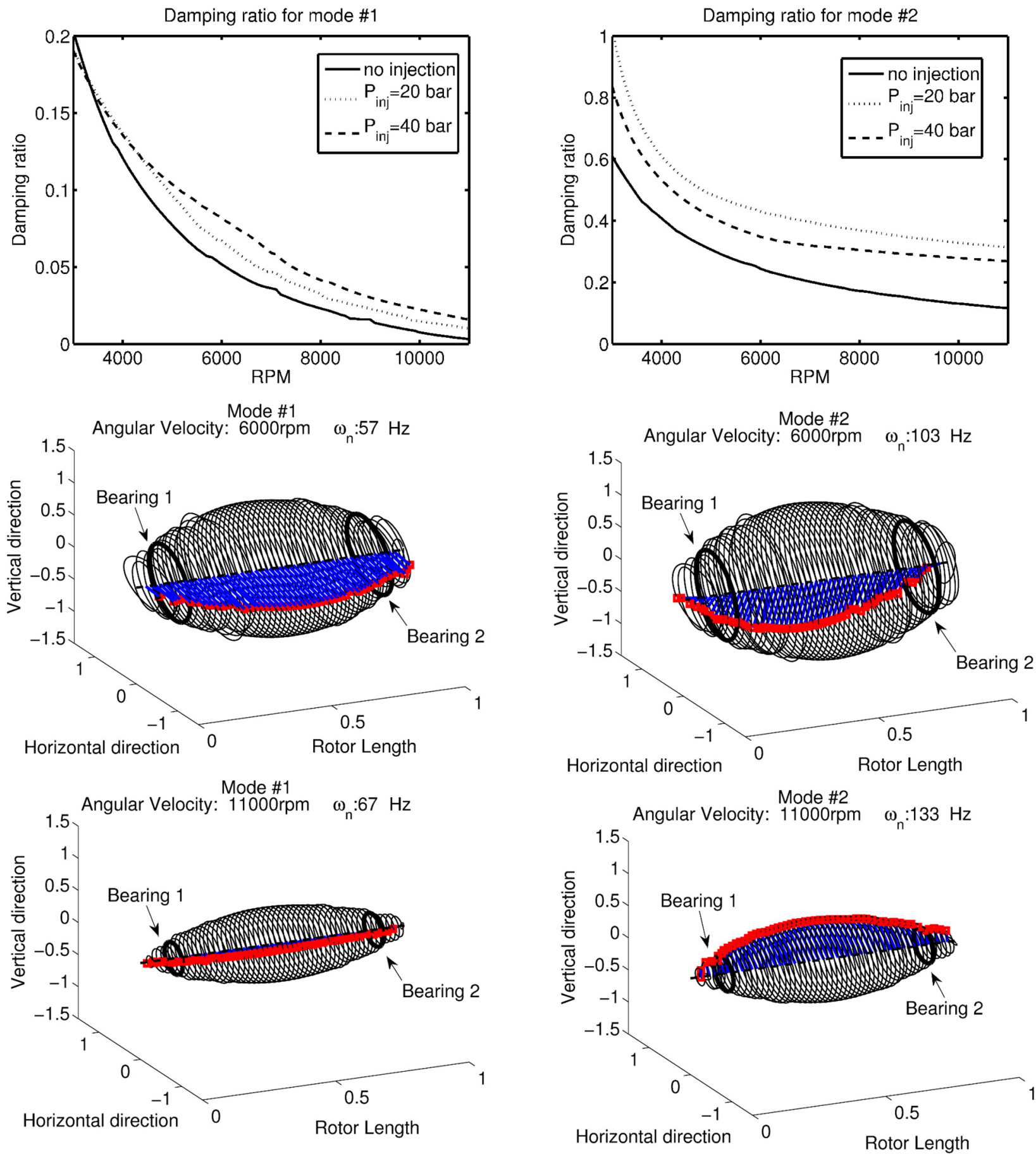

Fig. 11. Effect of the injection pressure over the damping ratio for two different modes of the rotor-bearings system; mode 1 corresponds to the mode approaching instability on (Fig. 10), whereas mode 2 is the mode excited on (Fig. 9).

ing to study the effect of imposing a controllable lubrication regime. Based on the results presented on this work, it is possible to conclude the following:

1. The developed ETHD model compares well to experimental and theoretical results from the literature for the passive tilting-pad journal bearing operating in laminar regime. From the validation, it is clear that improvements are possible in the form of improving the modellling of the heat transfer taking place between the oil film and the pad. On this regard, an investigation on the way of doing it will be performed, since the absence of the radial direction as part of the oil film modeling domain does not allow to apply the usual boundary conditions to include such effect.

2. The theoretical results obtained when analyzing a rotor tilting pad journal bearings system showed some clear benefits of the implementation of a controllable 
lubrication regime. When injecting oil through a hole located towards the leading edge of the pads, a reduction in the oil film average temperature was observed, as well as an improvement in the damping ratio characteristics of the rotor-bearing system, expressed in the form of reduced unbalance response when crossing critical speeds and higher instability onset speed.

3. Among the drawbacks of imposing the controllable lubrication regime, a reduction in the minimum oil film thickness is observed, as well as an increase in the maximum temperature of the oil film. This last result could be a consequence of the adiabatic assumption for the oil film thermal behavior and the non-inclusion into the model of heat transfer effects between the bearing pads and the surroundings.

4. With regards to the selection of the injection pressure, the results obtained show that the resulting modification of the bearing stiffness and damping characteristics affect the damping ratio of the rotor-bearing system eigenmodes in different ways. Hence, finding an optimum value for a fixed injection pressure that enables to reduce the unbalance response at any running speed proves to be a difficult task. This difficulty can be overcome by establishing an active lubrication regime, where the injection pressure is determined by means of a control loop, as it has been shown in other works in the subject.

5. An experimental validation of the ETHD model including the effect of the controllable lubrication regime is on the way, in order to prove the practical validity of the promising results of this theoretical study.

\section{References}

[1] J. Lund, Spring and damping for the tilting pad journal bearings, ASLE Trans. 7 (1964) 342-352

[2] P. Allaire, J. Parsell, L. Barrett, A pad perturbation method for the dynamic coefficients of tilting-pad journal bearings, Wear 72 (1981) 29-44

[3] J. Kim, A. Palazzolo, R. Gadangi, Dynamics characteristics of tehd tilt pad journal bearing simulation including multiple mode pad flexibility, ASME J. Vib. Acoust. 117 (1995) 123-135

[4] M. Fillon, D. Souchet, J. Frêne, Influence of bearing element displacements on thermohydrodynamic characteristics of tilting-pad journal bearings, Proc. Japan International Tribology Conference, Nagoya, 1990, pp. $635-640$

[5] I.F. Santos, Design and evaluation of two types of active tilting pad journal bearings, in: the active control of vibration, C.R. Burrows, P.S. Keogh (eds.) Mechanical Engineering Publications Limited, London, England, 1994, pp. $79-87$
[6] I.F. Santos, Strategien fur die erhohung der dampfungsreserve von kippsegmentgelagerten rotorsystemen, in: Schwingungen in rotierenden Maschinen, H.S.H. Irretier, R. Nordmann (éd.), Vieweg Verlag, Braunschweig, Germany, 1995, Vol 3, pp. 3-12

[7] I.F. Santos, Aktive Schmierung zur Regelung von Rotorsystemen, In: Schwingungen in rotierenden Maschinen, H.S.H. Irretier, R. Nordmann, (éd.), Vieweg Verlag, Braunschweig, Germany, 1997, Vol 4, pp. 37-47

[8] I.F. Santos, F. Russo, Tilting-pad journal bearings with electronic radial oil injection, J. Tribol. ASME Trans. 120 (1998) 583-594

[9] I.F. Santos, A. Scalabrin, Control system design for active lubrication with theoretical and experimental examples, J. Eng. Gas Turbine. Power T. ASME 125

[10] A.M. Haugaard, I.F. Santos, Multi-orifice active tiltingpad journal bearings-harnessing of synergetic coupling effects, Tribol. Int. 43 (2010) 374-1391

[11] A.M. Haugaard, I.F. Santos, Stability of multi orifice active tilting-pad journal bearings, Tribol. Int. 43 (2010) $1742-1750$

[12] A. Cerda, I.F. Santos, Stability analysis of an industrial gas compressor supported by tilting-pad journal bearings under different lubrication regimes, ASME J. Eng. Gas Turbine. Power T. (in print)

[13] I.F. Santos, R. Nicoletti, THD analysis in tilting-pad journal bearings using multiple orifice hybrid lubrication, J. Tribol. ASME Trans. 121 (1999) 892-900

[14] I.F. Santos, R. Nicoletti, Influence of orifice distribution on the thermal and static properties of hybridly lubricated bearings, Int. J. Solids Struct. 38 (2001) 2069-2081

[15] A. Brooks, T. Hughes, Streamline upwind/PetrovGalerkin formulations for convection dominated flows with particular emphasis on the incompressible NavierStokes equation, Comput. Methods Appl. Mech. Eng. 32 (1982) 199-259

[16] H. Nelson, J. McVaugh, The dynamics of rotor-bearing systems using finite element, J. Eng. Ind. 98 (1976) 593600

[17] M. Fillon, J.C. Bligoud, J. Frêne, Experimental study of tilting-pad journal bearings - comparison with theoretical thermoelastohydrodynamic results, J. Tribol. ASME T. 114 (1992) 579-588

[18] S. Taniguchi, T. Makino, T. Takeshita, T. Ichimura, A thermohydrodynamic analysis of large tilting-pad journal bearing in laminar and turbulent flow regimes with mixing, J. Tribol. ASME Trans. 112 (1990) 542-550

[19] J. Kim, A. Palazzolo, R. Gadangi, TEHD analysis for tilting pad journal bearings using upwind finite element method, Tribol. Trans. 37 (1994) 771-783

[20] K. Brockwell, D. Kleinbub, W. Dmochowski, Measurement and calculation of the dynamic operating characteristics of the five shoe tilting-pad journal bearing, Tribol. Trans. 33 (1989) 481-492

[21] A.M. Haugaard, On controllable elastohydrodynamic fluid film bearings, Ph.D. Thesis, Technical University of Denmark, 2010 